\title{
Avaliação do Serviço de Referência em Triagem Neonatal para hipotireoidismo congênito e fenilcetonúria no Estado de Mato Grosso, Brasil
}

\author{
Evaluation of the Neonatal Screening Program \\ for congenital hypothyroidism and phenylketonuria \\ in the State of Mato Grosso, Brazil
}

Inês Stranieri', Olga Akiko Takano²

\section{RESUMO}

Objetivo: Avaliar o Serviço de Referência em Triagem Neonatal para hipotireoidismo congênito e fenilcetonúria no Estado de Mato Grosso. Métodos: Estudo transversal, utilizando-se dados

' Laboratório do Serviço de Referência em Triagem Neonatal, Hospital Universitário Júlio Müller (HUJM), Universidade Federal de Mato Grosso (UFMT), Cuiabá, MT, Brasil ${ }^{2}$ Departamento de Pediatria, Faculdade de Ciências Médicas, UFMT, Cuiabá, MT, Brasil
Correspondência para: Inês Stranieri

Av. Dr. Hélio Ribeiro, 101, ap. 701 Parque Eldorado

78048-250 - Cuiabá, MT, Brasil inestranieri@gmail.com

Recebido em 6/Jun/2008 Aceito em 15/Fev/2009 secundários dos exames realizados no período de janeiro de 2003 a dezembro de 2004. Resulltados: Foram feitos 66.337 testes de triagem com uma cobertura populacional inferior a $70 \%$. A prevalência de fenilcetonúria foi de 1:33.068 nascidos vivos, e de hipotireoidismo congênito foi de 1:9.448 nascidos vivos. Apenas $22 \%$ das amostras foram coletadas na idade recomendada; a maioria realizou o teste de triagem entre 8 e 30 dias de vida. A mediana da idade na coleta do teste foi de 12 dias. Verificou-se que o serviço teve dificuldades na reconvocação dos casos suspeitos e dificuldades financeiras na obtenção dos insumos laboratoriais. Conclusões: $A$ idade na coleta e o atraso na fase de confirmação diagnóstica foram os principais motivos para o atraso do início do tratamento dos casos detectados pelo serviço. Arq Bras Endocrinol Metab. 2009;53(4):446-52.

Descritores

Hipotireoidismo congênito; fenilcetonúria; triagem neonatal; avaliação de programas e projetos de saúde

\begin{abstract}
Objective: To evaluate the Reference Center for Neonatal Screening for congenital hypothyroidism and phenylketonuria for the State of Mato Grosso. Method: Cross-sectional study using secondary data of screening tests carried out from January 2003 to December 2004. Results: 66,337 exams were conducted with population coverage of less than $70 \%$. The prevalence of phenylketonuria was 1:33,068 live births and of congenital hypothyroidism was 1:9,448 live births. Only $22 \%$ of the samples were collected at the recommended ag, and most of the samples were collected between the ages of 8 and 30 days. The median age at collection was 12 days. It was observed that the service had difficulties in recalling suspected cases and financial difficulties in obtaining laboratorial reagents. Conclusions: The age at the time of collection and the delay at the diagnostic confirmation stage were the principal reasons for the delay in the initiation of treatment of the cases detected by the service. Arq Bras Endocrinol Metab. 2009;53(4):446-52.

Keywords

Congenital hypothyroidism; phenylketonuria; neonatal screening; program evaluation
\end{abstract}

\section{INTRODUCÃO}

A fenilcetonúria (PKU) é um erro inato no metabolismo do aminoácido fenilalanina (FAL). É uma doença genética, causada por uma mutação no gene que codifica a enzima, a fenilalanina hidroxilase, que é ativada no fígado e responde pela transformação da fenilalanina em tirosina $(1,2)$. A elevação da fenilalanina no sangue permite a sua passagem em quantidade excessiva 
para o sistema nervoso central (SNC), no qual o seu acúmulo tem efeito tóxico $(3,4)$. Relatou-se uma prevalência de PKU no Brasil de 1:15.839 nascidos vivos em 2001 e 1:24.780 nascidos vivos em 2002 (5).

$\mathrm{O}$ hipotireoidismo congênito (HC) é uma doença causada pela deficiência ou ausência da ação dos hormônios tireoidianos nos vários tecidos do organismo (6). O hormônio tireoidiano é essencial para a maturação e o funcionamento de diversos órgãos do corpo, principalmente do SNC e do tecido esquelético. As repercussões da deficiência nesses tecidos dependem da época de início, intensidade, duração ou ausência do hormônio e, sobretudo, da normalização de seus níveis pela reposição hormonal precoce. Como o desenvolvimento do SNC ocorre mais intensamente no primeiro ano de vida, a deficiência ou a ausência do hormônio tireoidiano nessa fase provocarão lesões neurológicas - irreversíveis, na maioria das vezes $(7,8)$. Tornam-se, portanto, essenciais o diagnóstico e a reposição hormonal precoces para prevenir o retardo mental nas crianças que apresentam o HC $(9,10)$. Segundo Carvalho e cols. (5), a prevalência do HC no Brasil foi de 1:3.694 nascidos vivos em 2001 e 1:3.804 nascidos vivos em 2002.

A triagem neonatal tem por objetivo a detecção precoce dos erros inatos do metabolismo e outras patologias assintomáticas no período neonatal. A maioria dessas doenças pode ser tratada com sucesso, desde que sejam diagnosticadas antes de manifestarem seus sintomas claramente, a ponto de serem identificados por pais e médicos (11). Assim, todos os recém-nascidos (RN) devem ser submetidos ao teste - idealmente entre o terceiro e o sétimo dias de vida (12). Por utilizar amostras de sangue coletadas do calcanhar do RN, o exame ficou popularmente conhecido como Teste do Pezinho (8).

No Brasil, o Ministério da Saúde criou, em 2001, o Programa Nacional de Triagem Neonatal (PNTN) com meta de cobertura universal e garantia de realização de todas as etapas, que vão desde a coleta até o tratamento e acompanhamento dos casos detectados. O PNTN foi organizado em três fases de implantação: Fase I - HC e PKU; Fase II - HC, PKU e hemoglobinopatias; Fase III - HC, PKU, hemoglobinopatias e fibrose cística (13).

O PNTN é executado pelos Serviços de Referência em Triagem Neonatal (SRTN) em cada Estado e no Distrito Federal, sendo as instâncias ordenadoras e orientadoras destinadas à operacionalização, à execução e ao controle do PNTN em sua área de abrangência.

O Hospital Universitário Júlio Müller (HUJM) da Universidade Federal de Mato Grosso (UFMT) foi ca- dastrado como SRTN pela Portaria SAS/MS n ${ }^{\circ} .684$ de 4/10/2002. Em 2003, o serviço iniciou a Fase I.

Prevenir a deficiência mental é uma questão social relevante. A realização de um exame simples, como o Teste do Pezinho, é um importante instrumento de diagnóstico e prevenção de doenças genéticas e metabólicas, desde que seja realizado nos primeiros dias de vida do $\mathrm{RN}(14,15)$. Considerando-se a importância da deteç̧ão precoce da PKU e do HC, este estudo teve como objetivo avaliar o SRTN de Mato Grosso.

\section{MÉTODOS}

Trata-se de um estudo transversal, com a utilização de dados secundários do único SRTN de Mato Grosso, localizado no HUJM/UFMT.

A população de estudo foi composta por todos os nascidos vivos no período de janeiro de 2003 a dezembro de 2004 e que realizaram o teste de triagem na rede pública do Estado de Mato Grosso. Foram analisados todos os resultados das dosagens de fenilalanina e do hormônio tireoestimulante (TSH) de amostras de sangue coletadas em papel-filtro (Schleicher \& Schuell, 903).

No ano de 2003, as dosagens de FAL e TSH foram realizadas pelo método enzimático-colorimétrico, utilizando-se o equipamento Coda (Biorad $\left.{ }^{\circledR}\right)$. Em 2004, mudou-se para o método imunofluorimétrico, utilizando-se os equipamentos Vitore AutoDelphia (Perkin Elmer ${ }^{\circledR}$ ).

A cobertura do programa foi calculada pela razão do número de $\mathrm{RN}$ que realizaram o teste de triagem dividido pelo número de nascidos vivos no período estudado multiplicado por 100.

Foram calculadas as seguintes variáveis:

1. Idade média na coleta para o total de $\mathrm{RN}$ triados, para verificar se a coleta ocorria na idade ideal, entre o terceiro e o sétimo dias de vida.

2. Tempo médio gasto no transporte das amostras ao SRTN-MT, para verificar se o tempo excedia o prazo máximo de cinco dias, normatizado pelo Ministério da Saúde.

3. Tempo médio decorrido em dias para a emissão dos resultados pelo SRTN-MT, para verificar a capacidade de processamento do laboratório especializado em liberar os resultados no prazo máximo de cinco dias.

4. Idade das crianças na liberação do resultado, para verificar se os resultados eram liberados até a idade máxima recomendada para início do tratamento. 
5. Tempo médio de reconvocação dos casos positivos detectados, para verificar a agilidade na localização dos RN que precisam realizar novos procedimentos laboratoriais ou ambulatoriais.

6. Idade média dos $\mathrm{RN}$ ao diagnóstico, para verificar o tempo gasto no laboratório para a confirmação diagnóstica.

7. Idade média na primeira consulta, para verificar se o tratamento foi instituído no primeiro mês de vida do RN.

8. Número médio de consultas para verificar a assiduidade dos pacientes ao serviço.

Pela característica geográfica do Estado de Mato Grosso, a distância dos municípios e a dificuldade de acesso até a capital, nem sempre a idade dos $\mathrm{RN}$ ao diagnóstico confirmados laboratorialmente (item 5) corresponde à idade dos $\mathrm{RN}$ na primeira consulta (item 6).

Foi calculada a prevalência das patologias triadas para o número de $\mathrm{RN}$ investigados pela rede pública do Estado.

Os dados foram descritos por meio de frequências absolutas e relativas para as variáveis categóricas, e de médias e desvios-padrão para as variáveis numéricas contínuas. $\mathrm{Na}$ análise dos dados, utilizou-se o pacote estatístico Statistical Package for the Social Sciences (SPSS) versão 10.0.

\section{Considerações éticas}

Esta pesquisa foi submetida e aprovada pelo Comitê de Ética em Pesquisa do HUJM/UFMT, protocolo 347/ CEP-HUJM/05.

\section{RESULTADOS}

Foram realizados, pelo SRTN-MT, nos anos de 2003 e $2004,31.703$ e 34.634 testes de triagem, respectivamente.

Segundo o Sistema de Informação de Nascidos Vivos, foram registrados, em Mato Grosso, 48.539 nascimentos no ano de 2003, e 51.205 em 2004. Calculouse, a partir desses dados, uma cobertura populacional referente ao teste de triagem de $65,3 \%$ em 2003 e de $67,6 \%$ em 2004, caracterizando-se um discreto incremento desta dentre os anos avaliados.

Em 2003, a cobertura da rede de coleta do SRTNMT, nos 139 municípios do Estado, foi de aproximadamente $90 \%$, apresentando médias mensais de 2.642 testes $(\mathrm{DP}=326,6)$. Em 2004, a cobertura foi de aproximadamente $90,7 \%$, apresentando médias mensais de 2.848 testes $(\mathrm{DP}=437,0)$.

Foram identificados dois casos de PKU em 2003 e nenhum em 2004. Quanto ao HC, foram diagnosticados três casos em 2003 e quatro em 2004. Na população avaliada, as prevalências de PKU e HC, no período estudado, foram de 1:33.068 e 1:9.448 nascidos vivos triados, respectivamente.

Observou-se que, para a maioria dos $\mathrm{RN}(63 \%$; n $=19.960)$, a coleta da amostra de sangue para o teste de triagem foi realizada entre o $8^{\circ}$ e $30^{\circ}$ dias após o nascimento. No ano de 2004, maior percentual de coleta também foi realizado nessa faixa etária $(66,2 \% ; n$ $=22.934)$ (Tabela 1$)$. Verificou-se que um percentual considerável de indivíduos, tanto em 2003 como em 2004, teve amostras coletadas em idade superior a 30 dias, 9,8\% e 9\%, respectivamente (Tabela 1 ).

Tabela 1. Distribuição das crianças triadas, segundo a faixa etária (dias), na coleta do teste de triagem, Mato Grosso, 2003 e 2004

\begin{tabular}{ccccc}
\hline \multirow{2}{*}{$\begin{array}{c}\text { Faixa etária } \\
\text { (dias) }\end{array}$} & \multicolumn{2}{c}{$\mathbf{2 0 0 3}$} & \multicolumn{2}{c}{$\mathbf{2 0 0 4}$} \\
\cline { 2 - 5 } & $\mathbf{n}$ & $\mathbf{\%}$ & $\mathbf{n}$ & $\mathbf{\%}$ \\
\hline 0 a 2 & 572 & 1,8 & 501 & 1,4 \\
3 a 7 & 7.005 & 22,1 & 7.801 & 22,6 \\
8 a 30 & 19.960 & 62,9 & 22.934 & 66,3 \\
> 30 & 3.098 & 9,8 & 3.098 & 9,0 \\
Ignorados & 1.068 & 3,4 & 249 & 0,7 \\
Total & 31.703 & 100 & 34.583 & 100 \\
\hline
\end{tabular}

Foram reconvocados para uma nova coleta de amostras, durante os anos de 2003 e 2004, 437 (1,4\%) e 741 $(2,1 \%) \mathrm{RN}$, respectivamente. Amostra inadequada para análise foi a causa mais frequente de reconvocação.

Os valores encontrados dos indicadores para os anos de 2003 e 2004 no SRTN estão apresentados na Tabela 2. Comparando-se esses dados, em 2004 não se observou redução da idade média na coleta; houve aumento no tempo médio gasto no transporte e na realização dos exames, além de demora na emissão e liberação dos resultados.

Os dados dos casos diagnosticados de PKU e HC encontram-se descritos na tabela 3 .

Em 2003, foram realizadas 39 consultas para portadores de HC e 17 consultas para portadores de PKU. Em 2004, foram realizadas 28 consultas para portadores de PKU e 49 consultas para portadores de HC. 


\begin{tabular}{lcccccccc}
\hline Tabela 2. Indicadores das etapas do Programa de Triagem Neonatal em Mato Grosso, $2003(\mathrm{n}=31.703)$ e $2004(\mathrm{n}=34.634)$ \\
\hline Indicadores & $\begin{array}{c}\text { Idade da criança na data da } \\
\text { coleta }\end{array}$ & $\begin{array}{c}\text { Tempo gasto no transporte } \\
\text { da amostra até o SRTN }\end{array}$ & $\begin{array}{c}\text { Tempo gasto para a } \\
\text { emissão dos resultados }\end{array}$ & $\begin{array}{c}\text { Idade das crianças na } \\
\text { liberação do resultado }\end{array}$ \\
\cline { 2 - 10 } & $\mathbf{2 0 0 3}$ & $\mathbf{2 0 0 4}$ & $\mathbf{2 0 0 3}$ & $\mathbf{2 0 0 4}$ & $\mathbf{2 0 0 3}$ & $\mathbf{2 0 0 4}$ & $\mathbf{2 0 0 3}$ & $\mathbf{2 0 0 4}$ \\
\hline Mediana (dias) & 12 & 12 & 9 & 10 & 16 & 46 & 46 & 75 \\
Média (dias) & 16,3 & 15,9 & 12,9 & 14,1 & 26,0 & 48,1 & 55,3 & 77,9 \\
Desvio-padrão & 15,8 & 14,6 & 13,7 & 13,7 & 24,5 & 17,0 & 32 & 25,7 \\
Valor mínimo (dias) & 0 & 0 & 0 & 0 & 1 & 1 & 9 & 16 \\
Valor máximo (dias) & 374 & 311 & 269 & 273 & 253 & 252 & 432 & 438 \\
Ignorados (n) & 1.068 & 249 & 922 & 208 & 366 & 769 & 738 & 848 \\
\hline
\end{tabular}

*SRTN: Serviço de Referência de Triagem Neonatal.

Tabela 3. Casos confirmados de fenilcetonúria $(n=2)$ e hipotireoidismo congênito $(n=7)$ na população estudada de nascidos vivos, segundo a procedência e indicadores estudados, Mato Grosso, 2003 a 2004

\begin{tabular}{|c|c|c|c|c|}
\hline Cidade & $\begin{array}{l}\text { Idade da criança na } \\
\text { data da coleta (dias) }\end{array}$ & $\begin{array}{l}\text { Tempo gasto para a } \\
\text { emissão dos resultados } \\
\text { (dias) }\end{array}$ & $\begin{array}{l}\text { Tempo gasto para a } \\
\text { confirmação dos } \\
\text { resultados (dias) }\end{array}$ & $\begin{array}{l}\text { Idade na } 1^{\mathrm{a}} \text { consulta } \\
\text { (dias) }\end{array}$ \\
\hline \multicolumn{5}{|l|}{ Fenilcetonúria (2003) } \\
\hline Araputanga & 6 & 11 & 30 & 48 \\
\hline Sorriso & 9 & 22 & 50 & 100 \\
\hline \multicolumn{5}{|c|}{ Hipotireoidismo congênito (2003) } \\
\hline Cuiabá & 21 & 14 & 7 & 47 \\
\hline Planalto da Serra & 32 & 40 & 16 & 93 \\
\hline Cuiabá & 100 & 3 & 22 & 126 \\
\hline \multicolumn{5}{|c|}{ Hipotireoidismo congênito (2004) } \\
\hline Cuiabá & 16 & 11 & 31 & 64 \\
\hline Cuiabá & 11 & 7 & 56 & 76 \\
\hline Campo Novo do Parecis & 14 & 15 & 42 & 82 \\
\hline Rondonópolis & 9 & 9 & 73 & 102 \\
\hline
\end{tabular}

\section{DISCUSSÃO}

Considerando-se que o SRTN-MT foi implantado em 2003 , a cobertura populacional abaixo de $70 \%$ para os dois primeiros anos revela um desempenho satisfatório. No período estudado, a cobertura da rede de coleta estadual não sofreu o incremento esperado e verificou-se a falta de implantação de postos de coleta em $12 \mathrm{mu}-$ nicípios. A cobertura populacional total é muitas vezes dificultada por problemas socioeconômicos e culturais, falta de informações da importância do teste de triagem neonatal e dificuldade dos pais - principalmente os que moram em zonas rurais - de acesso aos postos de coleta para a realização dos exames $(10,16)$.

Vários fatores dificultam o acesso aos Serviços de Saúde, principalmente dos municípios ao norte do Estado: a extensão territorial do Estado de Mato Grosso, a localização geográfica de alguns municípios com gran- de parte da população na zona rural, além das péssimas condições nas estradas nos períodos de chuva.

A implantação no Brasil de SRTN elevou a cobertura global estimada para aproximadamente $80 \%$ em 2005 (13), porém, de forma bastante heterogênea.

As maiores coberturas se encontram nos Estados credenciados para a Fase III, e o inverso se dá com aqueles credenciados para a Fase I. Cabe ressaltar que Mato Grosso (Fase I) e Mato Grosso do Sul (Fase II) apresentam coberturas discrepantes em relação aos outros Estados que se encontram nas mesmas fases. De uma forma geral, há uma expressiva heterogeneidade na distribuição das coberturas, que provavelmente tem relação com a situação dos indicadores de desenvolvimento econômico, social, político, cultural e de saúde (17).

A prevalência da PKU em Mato Grosso foi de 1:33.068 nascidos vivos na população estudada, menor 
que a encontrada para o Brasil por Carvalho e cols. (5) de 1:24.780 nascidos vivos.

A prevalência do HC foi de 1:9.448 nascidos vivos na população estudada, menor que a relatada por Carvalho (5) para o Brasil, de 1:3.804 nascidos vivos.

Botler e cols. (17) fizeram uma análise da triagem neonatal no mundo. Esses autores observaram que o desenvolvimento, na Europa, tem sido lento e heterogêneo; mais rápido nos países ocidentais; e mais lento no Leste Europeu - com cobertura de 69\% em 2004, em dados abrangendo 38 países. Essa heterogeneidade também foi observada na Ásia e na região do Pacífico, sendo que países dessa região, em que a taxa de mortalidade infantil é menor do que 10/1.000 nascidos vivos, tinham coberturas superiores a $90 \%$. Na América Latina, a cobertura foi de $49 \%$ em 2005 , com dados de 14 países. No Canadá, em 2006, a cobertura foi de 71\% em 2006. Segundo esses autores (17), os esforços do governo norte-americano e de organizações médicas, no sentido de promover o compartilhamento e a integração entre os sistemas de dados de saúde neonatal e os de triagem neonatal, levam a supor que a cobertura desse programa seja universal em todos os Estados da federação.

No Brasil, os autores também relataram a heterogeneidade de cobertura populacional regional notada por meio dos diferentes valores de cobertura alcançados pelos programas do Norte e Nordeste do país quando comparados aos programas do Sul e Sudeste (17-19).

A idade ideal preconizada para a coleta das amostras é de três a sete dias de vida $(3,18)$. A coleta acima de 30 dias de vida é inadequada e antes de 48 horas de vida é precoce, pois, nessas condições, podem ocorrer resultados falsos-negativos para PKU $(18,20)$.

A maior parte das crianças nesse estudo realizou a coleta no período entre 8 e 30 dias, considerada inaceitável para um programa que tem por objetivo o início precoce do tratamento das patologias triadas. Em 10\% dos RN a coleta foi feita após 30 dias de vida, idade superior à recomendada para a prevenção de sequelas, mostrando a necessidade de realizar um trabalho de divulgação e orientação com os pais e profissionais da Saúde, abordando-se a importância da faixa etária adequada para a realização do teste de triagem e sua relação com a prevenção das doenças triadas.

A demora no processamento dos exames pode ser justificável no Mato Grosso, pela prática de retenção das amostras colhidas, isto é, são acumuladas amostras coletadas em vários dias para posterior envio ao SRTN. Evidenciam-se dificuldades no transporte, consideran- do-se as grandes distâncias a serem percorridas, devido à localização geográfica dos municípios do interior do Estado e ao péssimo estado de conservação das estradas, principalmente no norte do Estado. No laboratório de triagem neonatal, a possível falta de reagentes para a realização dos exames, em 2004, acarretou demora para sua realização.

No período analisado, a principal causa de reconvocação dos $\mathrm{RN}$ foi a coleta de amostras inadequadas para análise, como: amostra insuficiente, hemolisada, em excesso, diluídas e com menos de 48 horas de vida. Os dados analisados indicam dificuldades na execução da técnica de coleta e armazenamento das amostras, que podem estar relacionadas à falta de treinamento técnico, ao desconhecimento da importância pelo profissional que realiza a coleta das amostras e à alta rotatividade de profissionais.

$\mathrm{Na}$ análise dos indicadores, nos casos confirmados de HC, verificou-se que, em 2003, havia problemas em relação à idade na coleta, sendo esse o principal fator para o retardo do início do tratamento. Quando comparados a 2004, houve melhora na idade da coleta, porém, houve piora considerável no tempo gasto para a confirmação do diagnóstico, fazendo com que permanecesse praticamente inalterada a idade da criança no início do tratamento. Isso indica que o serviço teve dificuldades no trabalho de reconvocação dos casos a serem confirmados, agravadas por dificuldades financeiras para a obtenção dos insumos laboratoriais, o que pode explicar a demora na realização dos exames.

No Brasil, há poucas publicações sobre a idade na coleta do teste e é possível observar que, nos Estados onde a cobertura se encontra mais longe da meta, as coletas são feitas mais tardiamente (Ceará, Paraíba, Bahia, Sergipe, Rio Grande do Sul e Espírito Santo). O oposto se observa nos Estados com melhor cobertura (Minas Gerais, Santa Catarina e Paraná). Talvez não por acaso os Estados em que a cobertura universal é acompanhada de menor tempo de coleta são aqueles que se encontram na Fase III do PNTN. Isso pode revelar uma melhor organização no acesso ao teste e, possivelmente, na estruturação dos respectivos programas. Os Estados com menor cobertura e tempos mais longos para a coleta se encontram nas Fases I e II, e esses dados sugerem a necessidade de maior investimento em medidas locais que facilitem o acesso ao teste, para que as coberturas se tornem, de fato, universais, e as coletas, oportunas (17).

Nos Estados Unidos, a Academia Americana de Pediatria (AAP) recomenda a coleta na alta hospitalar, sendo obrigatória uma segunda coleta em alguns Esta- 
dos, para melhorar a sensibilidade e especificidade do teste $(21,22)$.

Os resultados encontrados no SRTN-MT em relação ao atraso do diagnóstico e ao início do tratamento, tanto para os casos confirmados de HC como de PKU, apontam a necessidade de se desenvolver estratégias de melhoria da operacionalização, para promover uma maior agilidade em todo o processo e para que o início do tratamento ocorra em tempo adequado (23-26). A AAP e a Associação Americana de Tireoide afirmam que o tratamento instituído até 15 dias de vida é capaz de garantir o desenvolvimento neurológico normal, mesmo nos casos mais graves (26).

Estudo realizado em Pittsburgh demonstrou que o tratamento iniciado antes dos três meses resulta em quociente de inteligência médio de 89 , entre três e seis meses cai para 70 e, após os seis meses, apenas 54 (27).

Segundo Horovitz e cols. (28), o impacto dos defeitos congênitos no Brasil vem aumentando progressivamente, tendo passado da quinta para a segunda causa dos óbitos em menores de um ano, entre 1980 e 2000, fato que aponta para a necessidade de estratégias específicas na política de Saúde. Entre as ações localizadas no Brasil relacionadas aos defeitos congênitos, destacam-se os serviços do Programa de Triagem Neonatal.

A média de consultas por paciente foi superior à preconizada pelo Ministério da Saúde (quatro consultas por ano), demonstrando que, apesar das inúmeras dificuldades encontradas em todo o processo, a equipe multidisciplinar do SRTN-MT teve um bom desempenho no acompanhamento dos pacientes.

As limitações desse estudo foram representadas pela inexistência de dados referentes ao local de nascimento dos RN nos livros de registros, de dados na Secretaria de Estado de Saúde de Mato Grosso referentes às patologias estudadas e de um software adequado que gerenciasse os dados registrados no SRTN-MT. Essas limitações impossibilitaram a determinação da cobertura real do SRTN-MT por município e a comparação entre a prevalência das patologias, antes e após a sua implantação. Deste estudo resultou a construção de uma base de dados que favorecerá a gestão do Programa de Triagem Neonatal pelo SRTN-MT e oferecerá subsídios para análises posteriores, colaborando para a sistematização das informações.

É importante o estabelecimento de uma estratégia para sensibilização, informação e mobilização dos profissionais envolvidos em todo o processo de triagem neonatal, por meio de um programa de educação con- tinuada, visando permitir a uniformização dos procedimentos técnicos e maior conscientização dos papéis desempenhados por eles.

Para ampliar a cobertura populacional, há necessidade de produção e distribuição de material educativo em triagem neonatal para os profissionais de Saúde e para os usuários do Sistema Único de Saúde (SUS) do Estado de Mato Grosso. Poderiam ser aproveitadas as estruturas de outros programas, como o Programa Nacional de Imunização e o Pré-Natal, e, a exemplo de outros Estados, que divulgam o quinto dia de vida do RN para a coleta do Teste do Pezinho e o início da imunização.

Agradecimentos: aos funcionários do SRTN-MT pela colaboração e compreensão da importância deste trabalho.

Declaração: os autores declaram não haver conflitos de interesse científico neste artigo.

\section{REFERÊNCIAS}

1. Cederbaum S. Phenylketonuria: an update. Curr Opin Pediatr. 2002;14(6):702-6.

2. Martins AM. Inborn errors of metabolism: a clinical overview. São Paulo Med J. 1999;117(6):251-65.

3. Mira NV, Márquez UM. Importância do diagnóstico e tratamento da fenilcetonúria. Rev Saúde Pública. 2000;34(1):86-9.

4. Schmidt BJ, Martins AM, Fisberg RV, Andrade AAC, Subero EM. Fenilquetonuria: aspectos clínicos y terapêuticos. Pediatr Día. 1987;3(5):257-60.

5. Carvalho TM, Santos HP, Santos IC, Vargas PR, Pedrosa J. Newborn screening: a national public health programme in Brazil. $J$ Inherit Metab Dis. 2007;30:615.

6. Silva LO. Hipotireoidismo congênito transitório: perfil das crianças identificadas no programa estadual de triagem neonatal de Minas Gerais, Brasil. Arq Bras Endocrinol Metab. 2005;49(4):521-8.

7. LaFranchi S. Congenital hypothyroidism: etiologies, diagnosis and managements. Thyroid. 1999;9(7):735-40.

8. Januário JN, Mourão OG. Manual de organização e normas técnicas para triagem neonatal: programa estadual de triagem neonatal Minas Gerais. Belo Horizonte: Coopmed; 1998.

9. Bernal M, Caldas M, Bonilla R, Chamorro GA, Matallama A. Tamización para hipotiroidismo congênito em Cali y constituición de um centro de referencia para la identificación temprana de la enfermedad. Colomb Med. 2003;34(1):40-6.

10. Vulsma T, Kok JH. Prematurity-associated neurologic and developmental abnormalities and neonatal thyroid function. N Engl J Med. 1966;334(13):857-8.

11. Nascimento ML, Pires MMS, Nassar SM, Ruhland L. Avaliação do programa de rastreamento neonatal para hipotireoidismo congênito da Secretaria de Estado da Saúde de Santa Catarina. Arq Bras Endocrinol Metab. 2003;47(1):75-81.

12. Ramos AJS, Rocha AM, Costa ADM, Benicio AVL, Ramos ALC, Silva $C R A$, et al. Avaliação do programa de rastreamento de doenças congênitas em Campina Grande PB, Brasil. Arq Bras Endocrinol Metab. 2003;47(3):280-4.

13. Carvalho TM. Triagem neonatal no Brasil. Rev Méd Minas Gerais. 2005;15(Suppl.1):20-2. 
14. Silva MBGM, Zagonel IPS, Lacerda MR. A enfermagem na triagem neonatal. Acta Sci Health Sci. 2003;25(2):155-61.

15. Almeida AM, GodinhoTM, Teles MS, Rehem APP, Jalil HM, Fukuda TG, et al. Avaliação do Programa de Triagem Neonatal na Bahia no ano de 2003. Rev Bras Saúde Mater Infant. 2006;6(1):85-91.

16. Ramalho RJR, Ramalho ARO, Oliveira CRP, Oliveira MHA. Evolução do Programa de Triagem Neonatal para o hipotireoidismo congênito e fenilcetonúria no Estado de Sergipe de 1995 a 2003. Arq Bras Endocrinol Metab. 2004;48(6):890-6.

17. Botler J, Camacho LAB, Cruz MM, George P. 0761/2007 - Triagem Neonatal - O Desafio de uma Cobertura Universal Efetiva. Ciênc. saúde coletiva [periódico na internet] no prelo. [citado $2008 \mathrm{Dez}$ 08]. Disponível em: http://www.abrasco.org.br/cienciaesaudecoletiva/ artigos/artigo_int.php?id_artigo=2835.

18. Brasil. Ministério da Saúde. Editora do MS. Manual de Normas Técnicas e Rotinas Operacionais do Programa Nacional de Triagem Neonatal. Brasília; Ministério da Saúde; ago. 2004.

19. Nesi França S, Domingos MT. Triagem neonatal do hipotireoidismo congênito: novas conquistas... novos desafios... Arq Bras Endocrinol Metab. 2008;52(4):579-80.

20. Goldbeck AS, Pereira CT, Moreira MDG, Silveira EL, Vargas PR. Avaliação da cobertura de triagem neonatal e incidências das patologias triadas no Rio Grande do Sul. Rev Med Minas Gerais. 2003;13(Supl 2):97-9.

21. American Academy of Pediatrics AAP Section on Endocrinology and Committee on Genetics, American Thyroid Association Com- mittee on Public Health. Newborn screening for congenital hypothyroidism: recommended guidelines. Pediatrics. 1993;91:1203-9.

22. Therrell BL, Adams J. Newborn screening in North America. J Inherit Metab Dis. 2007;30(4):447-65.

23. Ramalho ARO, Ramalho RJR, Oliveira CRP, Santos EG, Oliveira MCP, Oliveira MHA. Programa de Triagem Neonatal para hipotireoidismo congênito no nordeste do Brasil: critérios e diagnósticos. Arq Bras Endocrinol Metab. 2008;52(4):617-27.

24. Ruiz BH, Der Parsehian S, Tovo A, Marcev F. Incidencia de hipotiroidismo congénito y fenilcetonuria en una población de recién nacidos vivos de un hospital perinatológico del Gobierno de la Ciudad de Buenos Aires. Años 2000-2004. Rev Hosp Mat Inf Ramón Sardá. 2005;24(1):20-2.

25. Botler J. Repercussões neurológicas do hipotireoidismo congênito. Arq Bras Endocrinol Metab. 1996;40(4):264-70.

26. American Academy of Pediatrics, Rose SR; Section on Endocrinology and Committee on Genetics, American Thyroid Association, Brown RS; Public Health Committee, Lawson Wilkins Pediatric Endocrine Society, et al. Update of newborn screening and therapy for congenital hypothyroidism. Pediatrics 2006;117:2290-303.

27. Klein $\mathrm{AH}$, Meltzer S, Kenny FM. Improved prognosis in congenital hypothyroidism treated before age three months. J Pediatr. 1972;81(5):912-3.

28. Horovitz DDG, Llerena Jr JC, Mattos RA. Atenção aos defeitos congênitos no Brasil: panorama atual. Cad Saúde Pública. 2005;21(4):1055-64. 